\title{
Effective atomic number and electron density determination using spectral X-ray CT
}

\author{
Busi, Matteo; Kehres, Jan; Khalil, Mohamad; Olsen, Ulrik Lund
}

Published in:

Anomaly Detection and Imaging with X-Rays (ADIX) IV

Link to article, DOI:

$10.1117 / 12.2519851$

Publication date:

2019

Document Version

Publisher's PDF, also known as Version of record

Link back to DTU Orbit

Citation (APA):

Busi, M., Kehres, J., Khalil, M., \& Olsen, U. L. (2019). Effective atomic number and electron density determination using spectral X-ray CT. In A. A., J. A. G., \& M. E. G. (Eds.), Anomaly Detection and Imaging with $X$-Rays (ADIX) IV (Vol. 10999). [1099903] SPIE - International Society for Optical Engineering. Proceedings of SPIE - The International Society for Optical Engineering https://doi.org/10.1117/12.2519851

\section{General rights}

Copyright and moral rights for the publications made accessible in the public portal are retained by the authors and/or other copyright owners and it is a condition of accessing publications that users recognise and abide by the legal requirements associated with these rights.

- Users may download and print one copy of any publication from the public portal for the purpose of private study or research.

- You may not further distribute the material or use it for any profit-making activity or commercial gain

- You may freely distribute the URL identifying the publication in the public portal 


\section{Effective atomic number and electron density determination using spectral x-ray CT}

\section{Matteo Busi, Jan Kehres, Mohamad Khalil, Ulrik L. Olsen}

Matteo Busi, Jan Kehres, Mohamad Khalil, Ulrik L. Olsen, "Effective atomic number and electron density determination using spectral x-ray CT," Proc. SPIE 10999, Anomaly Detection and Imaging with X-Rays (ADIX) IV, 1099903 (14 May 2019); doi: 10.1117/12.2519851

Event: SPIE Defense + Commercial Sensing, 2019, Baltimore, Maryland, United States 


\title{
Effective atomic number and electron density determination using spectral x-ray CT
}

\author{
Matteo Busi ${ }^{\mathrm{a}, \mathrm{b}}$, Jan Kehres ${ }^{\mathrm{a}}$, Mohamad Khalila ${ }^{\mathrm{a}}$, and Ulrik L. Olsen ${ }^{\mathrm{a}}$ \\ aTechnical University of Denmark, DTU Physics, Fysikvej 311, 2800 Kgs. Lyngby, Denmark
}

\begin{abstract}
We present Spectral X-ray Computed Tomography (SCT) estimations of material properties directly from energydependent measurements of linear attenuation coefficients (LAC). X-ray Computed Tomography (CT) is commonly utilized to characterize the internal properties of an object of interest. Dual-Energy X-ray CT allows material characterization into energy-independent physical properties such as $Z_{e}$ and electron density $\rho_{e}$. However, it is not robust in presence of dense materials and metal artifacts. We report on the performance of a method for system-independent characterization of materials that introduces a spectroscopic detector into X-ray $\mathrm{CT}$, called spectral $\rho_{e} / Z_{e}$ estimation (SRZE). We benchmark the SRZE method against energy-integrated measurements in material classification tests, finding superior accuracy in the predictions. The advantage of this technique, over other methods for material characterization using x-ray CT, is that it does not require a set of reference materials for calibration. Moreover, the simultaneous detection of spectral features makes it robust to highly attenuating materials, since the energy intervals for which the attenuation is photon limited can easily be detected and excluded from the feature estimation.
\end{abstract}

Keywords: Spectral X-ray CT, X-ray Characterization, Effective atomic number, Electron density, MULTIXME100, Threat Detection, Security Screening

\section{INTRODUCTION}

For decades, X-ray Computed Tomography (CT) has been adopted as one of the standard techniques for nondestructive tests and evaluations, for example in the screening of luggage for threats. ${ }^{1}$ Since laboratory-scale $\mathrm{X}$-ray sources generate a polychromatic beam (Fig. 1), conventional CT does not measure a single-energy value of the linear attenuation coefficient (LAC), but measures but its weighted average through multiple energies. Therefore, using this technique the characterization of a material's LAC is complicated by polychromatic effects such as beam hardening ${ }^{2}$ and photon starvation from metals or dense materials, ${ }^{3}$ and require an accurate model of the detector's spectral response. Moreover, different materials yield different contrast, in terms of intensity values in the reconstructions, when probed at different mean energies; therefore their classification might be not optimized depending on the choice of the source parameters of kilovoltage peak and filtration.

To overcome these limitations, researchers have recently developed methods to estimate energy-independent physical parameters of the materials, using Dual-Energy CT (DECT) ${ }^{4,5}$ With a DECT acquisition, the LAC of materials is probed at two different X-ray spectra (as the low- and high- energy pair in Fig. 1) and converted into the energy-independent physical parameters of electron density $\rho_{e}$ and effective atomic number $Z_{e}$, as in the System-Independent $\rho_{e} / Z_{e}$ (SIRZ) method proposed by Azevedo et al. ${ }^{6}$ and further developed by Champley et al. ${ }^{7}$ This can be technically achieved, for example with a consecutive scan with different source filtration and kilovoltage peaks, or using dual-energy sandwich detectors. ${ }^{8}$ However, the performance of these techniques depend on the choice of the pair of spectra used for the DECT acquisitions and the estimation of their respective detector spectral responses. These techniques will be accurate and precise for a group of materials within a limited range of $Z_{e}$, but will have lower performance for materials outside this range. This limitation can be overcome using spectral X-ray CT (SCT). SCT can be considered as an extension of DECT where the signal is simultaneously collected in multiple non-overlapping energy ranges. This would extend the range of materials for which the characterization is accurate.

Further author information: (Send correspondence to Matteo Busi)

Matteo Busi: E-mail: mbusi@fysik.dtu.dk, Telephone: +45 71706090

Anomaly Detection and Imaging with X-Rays (ADIX) IV, edited by Amit Ashok,

Joel A. Greenberg, Michael E. Gehm, Proceedings of SPIE Vol. 10999, 1099903

(c) 2019 SPIE $\cdot$ CCC code: $0277-786 X / 19 / \$ 18 \cdot$ doi: $10.1117 / 12.2519851$

Proc. of SPIE Vol. 10999 1099903-1 
With the advent of single photon counting detectors (PCD) able to discriminate the energy of the detected photons (Fig. 1), the interest towards spectral X-ray CT (SCT) has grown significantly, for the superior contrast to noise ratio ${ }^{9-13}$ and for enabling k-edge CT. ${ }^{14,15}$ In this work we use an extension of the SIRZ method for SCT, called Spectral $\rho_{e} / Z_{e}$ Estimation (SRZE), ${ }^{16}$ to estimate the physical parameters of a large dataset of innocuous materials commonly found in checked-in luggage, and threat materials which we want to be able to detect. The SRZE characterization's impact on the classification task accuracy is benchmarked against more direct methods for a set of different samples with including both innocuous and threat materials in a random order. For all cases studied in this work, we found superior accuracy in the classification task when using SRZE method for material characterization.
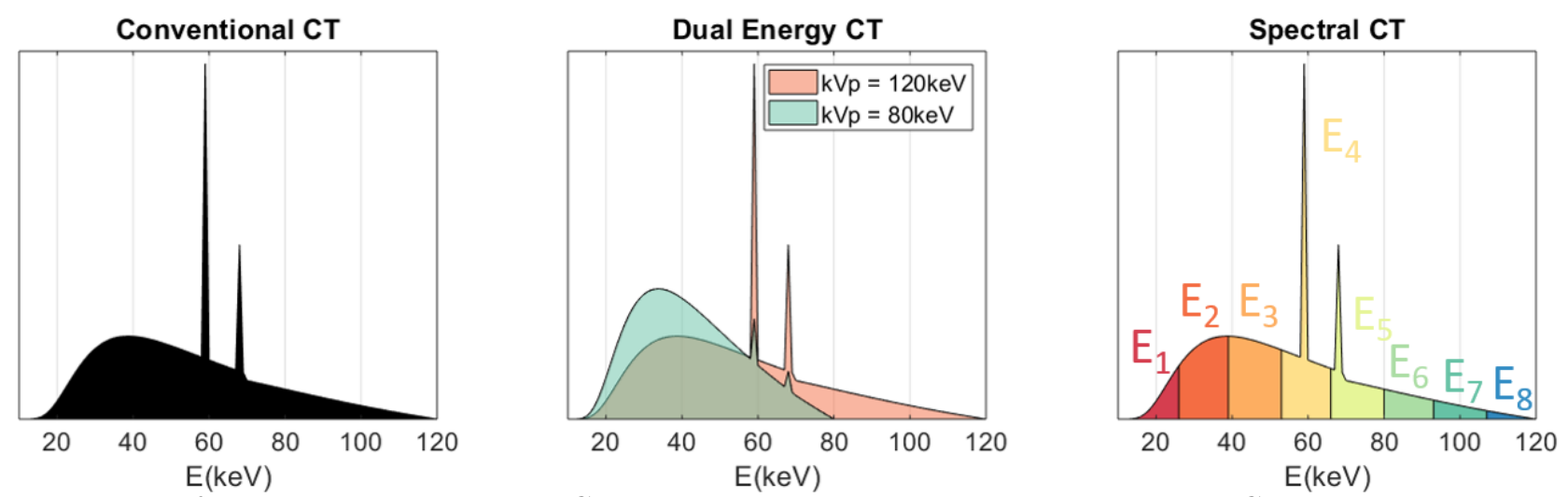

Figure 1: Left. In conventional X-ray CT, the signal is integrated over all energies. Center. In Dual-Energy $\mathrm{X}$-ray $\mathrm{CT}$, two different spectra are used to probe the sample attenuation at a low- and high- mean energy. Right. In Spectral X-ray CT, single photon counting detectors discriminate the incoming radiation in distinct energy bins with mean energy depending on the set energy thresholds.

\section{THEORY AND METHODS}

In this section, we define the system-independent physical properties we have used to characterize materials and the methods we have adopted in this work to estimate them from SCT measurements.

\subsection{Dual-Energy X-ray CT Characterization}

As described in the Lambert-Beer's law, ${ }^{17}$ X-ray CT measures the Linear Attenuation Coefficient (LAC) of materials. However, LAC is a function of the energy and therefore conventional CT, which use acquisitions with energy integrating detectors, is not a robust method for the characterization of materials since it depends on the source spectrum and polychromatic effects such as beam hardening that cause distortions in the reconstruction. Alvarez and Macovski showed in 1976 that the LAC could be approximated as linear combination of a dual set of basis functions: ${ }^{4}$

$$
\mu(E)=a_{1} f_{\text {ph.abs. }}(E)+a_{2} f_{K N}(E),
$$

where $f_{\text {ph.abs. }}(E)=1 / E^{3}$ models the photoelectric absorption interactions of photons with matter, and $f_{K N}(E)$ is the Klein-Nishina function, which approximately models the incoherent (Compton) scattering interactions of photons with matter:

$$
f_{K N}\left(E=E^{\prime} / 511 \mathrm{keV}\right)=\frac{1+E}{E^{2}}\left[\frac{2(1+E)}{1+2 E}-\frac{1}{E} \ln (1+2 E)\right]+\frac{1}{2 E} \ln (1+2 E)-\frac{1+3 E}{(1+2 E)^{2}} .
$$

The coefficients $a_{1}$ and $a_{2}$ are proportional to the physical parameters of bulk density $\rho$ and atomic number $Z$, which are energy-independent. Based on this concept, Azevedo et al. presented a method ${ }^{6}$ using Dual-Energy X-ray CT for the system-independent characterization of materials into their features of electron density $\left(\rho_{e}\right)$ 
and effective atomic number $\left(Z_{e}\right)$. For a compound material, consisting of a number $n_{i}$ of $N$ distinct elements $i$, the electronic density is defined as:

$$
\rho_{e}=\frac{\sum_{i=1}^{N} n_{i} Z_{i}}{\sum_{i=1}^{N} n_{i} A_{i}} \rho
$$

where $A(Z)$ is the atomic mass ( $\mathrm{g} /$ mole) of the element $\mathrm{Z}$. To approximate the atomic number of such a compound Azevedo et al. ${ }^{6}$ used the effective atomic number, $Z_{e}$, defined as a non-integer atomic number that corresponds to an artificial element, for which the interactions are assumed to be modeled by the X-ray attenuation cross sections. The cross sections for the artificial element $Z_{e}$ are obtained by a linear interpolation between the cross section of the two adjacent elements in the periodic table:

$$
\sigma_{e}\left(Z_{e}, E\right)=(1-\epsilon) \sigma_{e}\left(Z^{\prime}, E\right)+\epsilon \sigma_{e}\left(Z^{\prime}+1, E\right) .
$$

Therein, $Z^{\prime}$ is the lower adjacent element, $Z^{\prime}=\operatorname{floor}(Z)$ and $\epsilon$ is the difference, $\epsilon=Z^{\prime}-Z$. In terms of the effective atomic number and electronic density presented above, we express the LAC of a material $m$ as:

$$
\mu^{m}(E)=\rho_{e}^{m} \sigma_{e}\left(Z_{e}^{m}, E\right) .
$$

In their study, Azevedo et al. ${ }^{6}$ demonstrated system-independence of the method and very good accuracy and precision for a set of standard materials with $Z_{e}<15$ with well-known physical parameters.

\subsection{Spectral X-ray CT Characterization}

We briefly describe the method presented in detail by Busi et al. ${ }^{16}$ to estimate the effective atomic number and electron density from SCT acquisitions. The measured raw data undergoes a spectral detector correction algorithm presented by Dreier et al., ${ }^{18}$ which corrects for the spectral distortions caused by the interactions between photons and the PCDs. This step is followed by the spectral scattering corrections method, from Monte Carlo X-ray simulations, presented by Busi et al. ${ }^{19}$ The energy resolved sinograms are converted into attenuation using Lambert-Beer's law, and reconstructed using Livermore Tomography Tools (LTT) software ${ }^{20}$ using the filtered back projection algorithm. The energy-dependent LACs are obtained by taking the mean values in the manually segmented regions respective to each material, for each energy bin. The respective variance of the mean values in the segmented regions is calculated as well, as it will be used in the feature estimation step. At this stage, an automated energy thresholding is used to disregard energy bins in which the reconstructions are corrupted by photon starvation caused by highly dense materials. The estimation of the physical parameters is performed by solving a constrained minimization of the squared distance between the measured LAC, $\tilde{\mu}(E)$, and its relative theoretical definition $\mu(E)$, parametrized by $\rho_{e}$ and $Z_{e}$ as in eq. (5):

$$
\underset{\left\{\rho_{e}, Z_{e}\right\}}{\arg \min } \sum_{k=i}^{f} \lambda_{E_{k}}\left|\tilde{\mu}\left(E_{k}\right)-\rho_{e} \sigma_{e}\left(Z_{e}, E_{k}\right)\right|^{2} .
$$

Therein, $\lambda_{E_{k}}$ are the energy weights, computed as the reciprocal of the variance $\left(s^{2}\right)$ of each material's LAC in their ROI, $\lambda_{E_{k}}=1 / s^{2}\left(\tilde{\mu}_{E_{k}}\right)$. These can be interpreted as the weight given to each energy bin $E_{k}$ into the estimation of the material's features. The minimization process in this work was performed with MATLAB ${ }^{\circledR}$ 's routine function FMINCON with solution boundaries $\rho_{e}=0-15 \mathrm{e}^{-} \mathrm{mol} / \mathrm{cm}^{3}$ and $Z_{e}=1-99$ and initial guesses $\rho_{e}^{0}=1 \mathrm{e}^{-} \mathrm{mol} / \mathrm{cm}^{3}$ and $Z_{e}^{0}=7$. No particular dependency on the starting values was observed.

\section{EXPERIMENTS}

\subsection{Instrumental setup}

The instrumentation used for the characterization of the materials into their physical properties was designed for SCT measurements and briefed in Table 1. The X-ray beam was generated by a Hamamatsu source with a tungsten anode and the acceleration voltage and filament current set to $160 \mathrm{kV}$ and $0.5 \mathrm{~mA}$, respectively. For these operating parameters the focal spot was $75 \mu \mathrm{m}$. The detector was made up of two 1D PCD Multix-ME100 ${ }^{21}$ modules, composed of $1 \times 128$ pixels of size $0.8 \times 0.8 \mathrm{~mm}^{2}$ and each with 128 energy bins of width $1.1 \mathrm{keV}$, evenly 
distributed between 20 and $160 \mathrm{keV}$. The energy resolution of the detector under high X-ray fluxes is $6.5 \%$ ( $8 \mathrm{keV}$ at $122 \mathrm{keV}) .{ }^{22}$ A $2-\mathrm{mm}$-thick aluminum filter was placed in front of the source to reduce the beam hardening effects and to suppress the photons with energy below the spectral range of the detector. The characteristic $\mathrm{X}$-ray peaks of the tungsten target are smeared due to the limited detection energy resolution, ${ }^{18,23}$ resulting in a smoother spectrum compared to what is theoretically expected. For all the SCT scans performed, the total acquisition time per scan was 30 minutes with the rotation stage continuously rotating. Each scan was rebinned into 5 seconds frames to form 360 projections evenly distributed between $0^{\circ}$ and $359^{\circ}$. The source to detector distance (SDD) was set to $1115 \mathrm{~mm}$ whereas the source to object distance (SOD) was set to $575 \mathrm{~mm}$.

Table 1: Experimental parameters.

\begin{tabular}{|l|r|}
\hline Source parameters & $160 \mathrm{keV}, 0.5 \mathrm{~mA}$ \\
Focal spot & $75 \mu \mathrm{m}$ \\
Number of pixels & $1 \times 2561 \mathrm{D}$ array \\
Pixel size & $0.8 \times 0.8 \mathrm{~mm}^{2}$ \\
Detector's resolution & $6.5 \%(8 \mathrm{keV})$ at $122 \mathrm{keV}$ \\
Number of energy bins & $128(1.1 \mathrm{keV}$ width $)$ \\
Number of projections & 360 \\
Exposure time per projection & 5 seconds \\
SDD & $1115 \mathrm{~mm}$ \\
SOD & $575 \mathrm{~mm}$ \\
\hline
\end{tabular}

\subsection{Characterization results}

We used the SCT characterization method described in Section 2.2 to estimate the physical parameters of a set of 73 different materials listed in Table 3 in Appendix A. The results are displayed in a $\rho_{e} / Z_{e}$ chart, in Fig 2. The choice of the materials in this work focus on the threat detection in airport luggage. It consists of a varied selection of organic innocuous compounds, mostly in water solutions, and a selection of explosive or precursor threat materials. We found a good contrast in the two physical parameters of $\rho_{e}$ and $Z_{e}$ for threat and innocuous materials, except for a few materials. Ethanol $96 \%$ (index: 25) for example, will have a high chance of being detected as a false positive for its proximity to acetone (index: 3). Among the threats, the hydrazine solution in water (index: 7) and nitromethane (index: 12) are also challenging, due to their similarity with water (index: 48).

\subsection{Singular Value Decomposition analysis}

We estimate material physical parameters from their LAC using the model in Eq. 5. To confirm that this model matches with the experimental findings, we performed a Singular Value Decomposition (SVD) analysis procedure, similar to the one proposed by Eger et al. ${ }^{24}$ for synthetic data. We constructed a matrix $\mathcal{M}$ with the linear attenuation coefficients measured of all the materials listed in Appendix A. The matrix $\mathcal{M}$ has dimensions $\left(E_{b} \times N_{\text {mat }}\right)$ where $E_{b}$ is the number of energy bins for which the LAC is probed and $N_{\text {mat }}$ is the number of materials. The SVD decomposition aims to find a set of linearly independent vectors that can express the LAC, and can be expressed as:

$$
\operatorname{SVD}(\mathcal{M}) \equiv M=U S V^{T},
$$

where $U$ is a $\left(E_{b} \times N_{\text {mat }}\right)$ matrix where each column $i$ compose the set of linearly independent vectors $U_{i}(E), S$ is a $\left(N_{\text {mat }} \times N_{\text {mat }}\right)$ diagonal matrix where the magnitude of each element $S_{i, i}$ describes the relative contribution of the corresponding vector $U_{i}$ to the model. The first singular values $S_{i, i}$ and the first six singular vectors $U_{i}$ are shown in Fig. 3. A qualitative look at these vectors indicate the two dominant singular vectors, $U_{1}$ and $U_{2}$ are corresponding to the basis functions for incoherent scattering and photoelectric absorption, as in Eq. 1. These observations were confirmed by finding a linear proportionality between $U_{1}$ and the Klein-Nishina function $f_{K N}$, and a linear trend of the inversion $1 / \sqrt[3]{U_{2}(E)}$. The remaining singular vectors are dominated by noise components and the oscillations in the measured LAC due to the poor spectral resolution of the detector around 

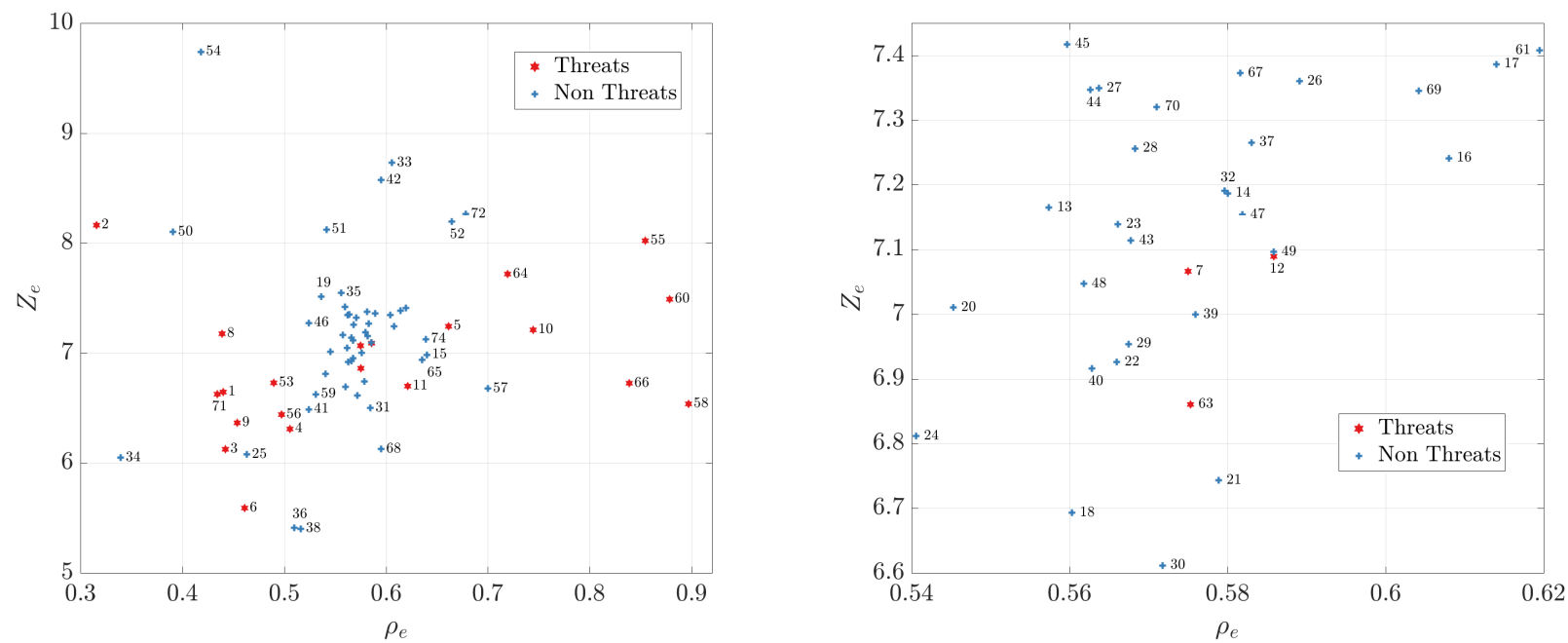

Figure 2: Charts of the effective atomic number and electron density $\left(Z_{e}, \rho_{e}\right)$ of the materials scanned, estimated through the SRZE method. The numbers labeling the data points are referring to the index in Table 3 in Appendix A. Note that to avoid overlap of the material labelled indexes, a magnified version of the left frame is displayed in the right frame.

the characteristic peaks of the X-ray source, as indicated by the wiggles around the energy of the $K_{\alpha_{1}}(59.3 \mathrm{keV})$ and $K_{\beta_{1}}(67.2 \mathrm{keV})$ tungsten emission lines. The third singular vector $U_{3}$, displays a sharp rise and consecutive relaxation, and is required to fully represent all the materials LACS. One would find the same behavior of the third significant singular vector, when running the same procedure on standard reference LAC curves from NIST database. ${ }^{25}$ We found, however, that the first two vectors are sufficient to approximate all the materials studied. This was confirmed by calculating the autocorrelation values of the singular vectors, which were below the value of $0.5^{26}$ except for $U_{1,2,3}$ and a few more $U_{4,5,6}$, as seen in Fig. 3 (b), with the same behavior as $U_{3}$ but different modulation of the peaks.

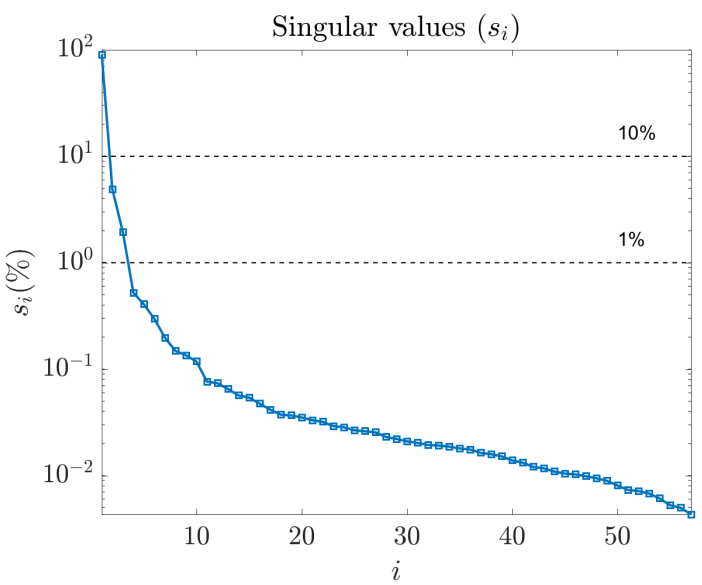

(a)
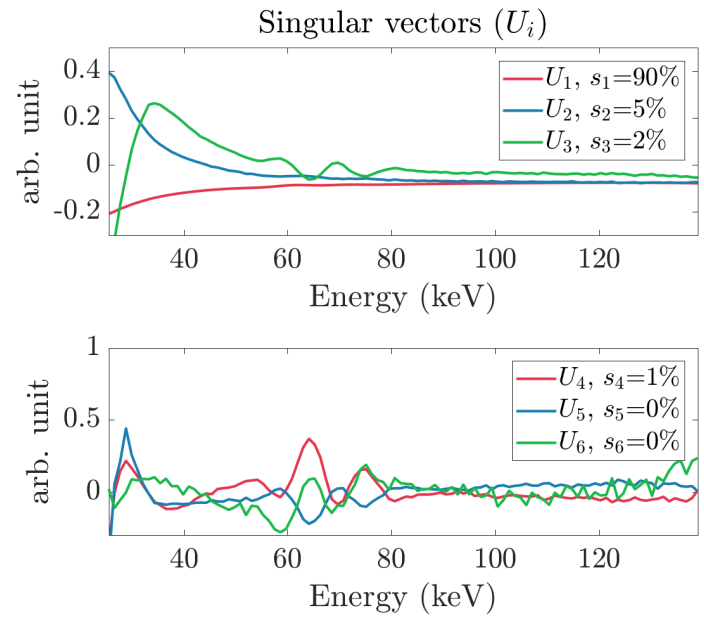

(b)

Figure 3: Singular Value Decomposition analysis of the reference materials LACs. (a) Singular values of the diagonal $i$-elements (blue-squares). Note logarithmic scale in the $y$-axis. (b) Singular vectors $U_{i}$ respective of the diagonal singular values $s_{i}$. The scale is in arbitrary units. 


\section{CLASSIFICATION BENCHMARKING}

\subsection{Materials and prediction models}

In this work, we have tested three different methods for the material classification from SCT data acquisitions. In the first method, we used as the material's feature the energy integrated LAC, $\tilde{\mu}\left(E^{\prime}\right)$, obtained by summing the signal over all the 128 energy channels of the detector. The effective energy $\left(E^{\prime}\right)$ corresponding to $\tilde{\mu}$ was calculated using the source spectrum as measured by the flat field acquisition (i.e. without the sample) as a model for the detector's response. The second method, utilizes as the material's feature the energy-dependent LAC, i.e. the attenuation coefficient probed at the 128 energy values corresponding to the specifics of the detector $\left(\mu\left(E_{k}\right)\right.$ with $k=1,2,3, \ldots, 128)$. In the third method, we used the SRZE characterization method described in Section 2. For each of these methods, a look-up table library of materials features such as the one in Appendix A, has been built by using single slice fan-beam SCT of individual materials in ideal condition (i.e. without metal artifacts and center-offset).

In the following scans, we have measured 30 samples composed of four glass bottles filled with different materials (found in Appendix A) and aluminum pin of $4 \mathrm{~mm}$ diameter placed in a sample holder. Of these samples, 10 of them were with the sample holder centered with the sample's rotation stage, 10 of them were with an offset between the sample holder and rotation stage centers, and the remainder 10 with an aluminum slab of $10 \times 30 \mathrm{~mm}$ as well. Examples of the CT reconstructions of the different groups are shown in Fig. 4 .
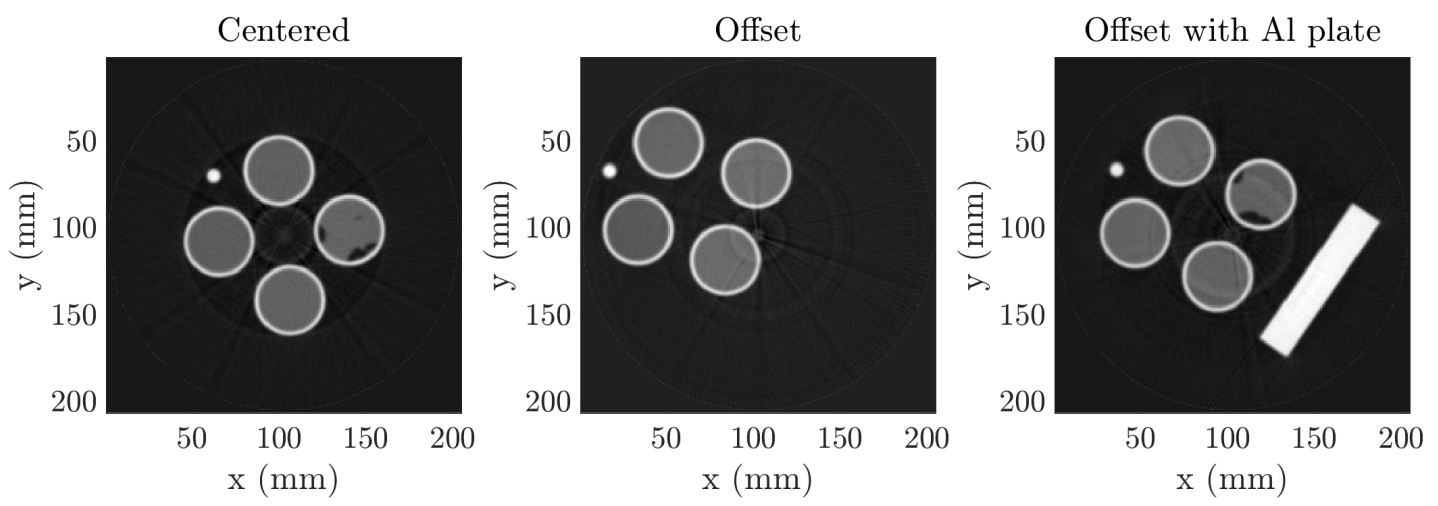

Figure 4: Reconstruction examples of the three types of dataset reproduced. Left: the samples are placed centered in respect to the center of rotation. Center: the samples are placed offset in respect to the center of rotation. Right: the samples are placed offset in respect to the center of rotation and an aluminum plate $10 \times 30 \mathrm{~mm}$ wide is inserted to provoke metal artifacts and photon starvation.

For each of the four materials in the samples, we have benchmarked the classification accuracy, as the ratio between successful predictions and total cases, of the three methods. The prediction models in the equations below have been used for the material classification to find the labels (i.e. the type of materials) from the ground truth library obtained using SRZE method listed in Table 3 in Appendix A. A similar library has been constructed for the other two methods.

- Integrated LAC discrimination:

$$
\text { label }=\underset{\text { label }}{\arg \min } \sqrt{\left(\mu^{e s t}-\hat{\mu}^{\text {label }}\right)^{2}} .
$$

- Spectral LAC discrimination:

$$
\text { label }=\underset{\text { label }}{\arg \min } \sqrt{\sum_{k=1}^{128}\left(\mu_{E_{k}}^{\text {est }}-\hat{\mu}_{E_{k}}^{\text {label }}\right)^{2}} .
$$


- SRZE discrimination:

$$
\text { label }=\underset{\text { label }}{\arg \min } \sqrt{w_{Z_{e}}\left(\frac{Z_{e}^{\text {est }}-\hat{Z}_{e}^{\text {label }}}{<\hat{Z}_{e}^{\text {label }}>}\right)^{2}+w_{\rho_{e}}\left(\frac{\rho_{e}^{\text {est }}-\hat{\rho}_{e}^{\text {label }}}{<\hat{\rho}_{e}^{\text {label }}>}\right)^{2}} .
$$

The hat sign refers to the values stored in the libraries whereas the $<>$ operator corresponds to the mean, applied to all reference values of $Z_{e}$ and $\rho_{e}$. That is done to have the physical parameters in the same scale and with same importance. The weights $w_{\rho_{e}}$ and $w_{Z_{e}}$ are assigned to the individual features to tune the significance given to each of them. In this work both have been set to $w=0.5$.

\subsection{Results}

The results of the benchmarking are reported in Table 2 for the different prediction methods and sample groups. In the material classification, the prediction is successful when the individual material label is found correctly,

Table 2: The accuracy results (\%) obtained for the different sample groups. In the exact material classification, the prediction is successful when the specific materials are classified correctly. In the binary classification, the discrimination is only performed between threats and innocuous materials.

Material classification

\begin{tabular}{|l|r|r|r|}
\hline & Integrated LAC $(\tilde{\mu})$ & Spectral LAC $(\mu(E))$ & SRZE $\left(\rho_{e} / Z_{e}\right)$ \\
\hline Centered & $24.0 \%$ & $72.0 \%$ & $72.0 \%$ \\
Offset & $28.0 \%$ & $68.0 \%$ & $72.0 \%$ \\
Offset with Al plate & $40.0 \%$ & $38.3 \%$ & $58.3 \%$ \\
Mean & $30.7 \%$ & $59.4 \%$ & $67.4 \%$ \\
\hline
\end{tabular}

Binary classification (Threat/Innocuous)

\begin{tabular}{|l|r|r|r|}
\hline & Integrated LAC $(\tilde{\mu})$ & Spectral LAC $(\mu(E))$ & SRZE $\left(\rho_{e} / Z_{e}\right)$ \\
\hline Centered & $60 \%$ & $100 \%$ & $100 \%$ \\
Offset & $64 \%$ & $98 \%$ & $100 \%$ \\
Offset with Al plate & $75 \%$ & $66.7 \%$ & $78.3 \%$ \\
Mean & $66.3 \%$ & $88.2 \%$ & $92.8 \%$ \\
\hline
\end{tabular}

whereas in the binary classification the discrimination is only done between innocuous and threat materials. Both the spectral characterization methods (LAC and SRZE) show an overall improved prediction accuracy when compared to integrated LAC method (which is corresponding to conventional integrating detectors). Samples with metal artifacts induced by the aluminum plate show a significant decrease in the accuracy of the direct LAC discrimination method, whereas the accuracy increases with the SRZE method. That is due to the energy thresholds selection step of the SRZE method, which allows data affected by photon starvation induced by the Aluminum plate to be disregarded. We remark that such a relatively low accuracy in the exact material classification was expected as most of the innocuous materials in the library are water-based compounds and some of them are varieties of the same specimens (e.g. 5 types of beer, 4 sun lotions etc.). On the other hand, the SRZE method shows perfect accuracy in the binary classification for samples without the aluminum plate. Nevertheless, the relatively low result obtained in the presence of metal artifacts suggests a demand for a further optimization of the data pre-processing step, possibly with the introduction of a metal artifact removal step.

\section{CONCLUSIONS}

We have demonstrated feasibility of material characterization into system-independent physical parameters $\left(\rho_{e}\right.$, $Z_{e}$ ) using spectral X-ray CT. We have shown that the spectral methods have overall superior accuracy in the material classification, when compared to the conventional CT techniques. Spectral LAC method shows a $28.7 \%$ and $21.9 \%$ increase in material and binary overall mean classification, respectively. The SRZE method shows a 
$36.7 \%$ and $26.5 \%$ increase in material and binary overall mean classification, respectively. The SRZE method is free of calibration steps and system-independent, which makes it suitable for a standard method estimation of a material's physical properties $\rho_{e}, Z_{e}$. Moreover, the SRZE method presents higher robustness in the presence of high-Z materials due to the capability to omit photon starved data. Further developments in the metal artifact removal algorithms for SCT, and advances in the hardware technologies of the PCD, decreasing their spectral distortions, should increase the screening of threats even further, using this method.

\section{APPENDIX A. MATERIALS ESTIMATED FEATURES LIBRARY}

The library of materials, for which the physical parameters were estimated, includes innocuous materials, which are commonly found in checked-in luggage, and explosive or precursor materials, which are aimed to be screened by the SCT inspection. Note that it is composed of mostly organic and liquid materials without a k-edge discontinuity and with $6 \leq Z_{e} \leq 10$. Table 3 provides a summary of all the materials scanned by themselves, their type and their estimated physical parameters, using the SRZE method described in Section 2. This table was used as the ground truth for material classification when the materials are scanned in groups of 4 .

Table 3: The list of all the 73 materials scanned and processed through the SRZE method, and their estimated physical parameters $\rho_{e}\left(\mathrm{e}^{-} \mathrm{mol} / \mathrm{cm}^{3}\right)$ and $Z_{e}$.

\begin{tabular}{|c|c|c|c|c|}
\hline Index & Material label & Type & $Z_{e}$ & $\rho_{e}$ \\
\hline 1 & 2-Butanone & Threat & 6.64 & 0.440 \\
\hline 2 & 2,4-Dinitrotoluene & Threat & 8.16 & 0.316 \\
\hline 3 & Acetone & Threat & 6.12 & 0.442 \\
\hline 4 & Ethylenediamine & Threat & 6.31 & 0.506 \\
\hline 5 & $\mathrm{H}_{2} \mathrm{O}_{2} 50 \%$ & Threat & 7.24 & 0.661 \\
\hline 6 & Hexamethylenetetramine & Threat & 5.59 & 0.461 \\
\hline 7 & Hydrazine solution & Threat & 7.07 & 0.575 \\
\hline 8 & Methanol & Threat & 7.17 & 0.439 \\
\hline 9 & N,N-Dimethylhydrazine & Threat & 6.36 & 0.454 \\
\hline 10 & Nitric acid $65 \%$ & Threat & 7.21 & 0.744 \\
\hline 11 & Nitrobenzene & Threat & 6.70 & 0.621 \\
\hline 12 & Nitromethane & Threat & 7.09 & 0.586 \\
\hline 13 & Wine & Innocuous & 7.16 & 0.557 \\
\hline 14 & Cream Liquor & Innocuous & 7.19 & 0.580 \\
\hline 15 & Balsamic Vinegar & Innocuous & 6.98 & 0.640 \\
\hline 16 & Bromhexin DAK & Innocuous & 7.24 & 0.608 \\
\hline 17 & Baby Shampoo & Innocuous & 7.39 & 0.614 \\
\hline 18 & Aftersun Lotion 1 & Innocuous & 6.69 & 0.560 \\
\hline 19 & Brandy & Innocuous & 7.51 & 0.537 \\
\hline 20 & Hand cream & Innocuous & 7.01 & 0.545 \\
\hline 21 & Sun Lotion 1 & Innocuous & 6.74 & 0.579 \\
\hline 22 & Body Lotion 1 & Innocuous & 6.93 & 0.566 \\
\hline 23 & Beer 1 (Wheat) & Innocuous & 7.14 & 0.566 \\
\hline 24 & Ethanol $40 \%$ & Innocuous & 6.81 & 0.541 \\
\hline 25 & Ethanol $96 \%$ & Innocuous & 6.08 & 0.463 \\
\hline 26 & Shampoo & Innocuous & 7.36 & 0.589 \\
\hline 27 & Beer 2 (Brown Ale) & Innocuous & 7.35 & 0.564 \\
\hline 28 & Porto Wine & Innocuous & 7.26 & 0.568 \\
\hline 29 & Aftersun Lotion 2 & Innocuous & 6.95 & 0.567 \\
\hline 30 & Sun Lotion 2 & Innocuous & 6.61 & 0.572 \\
\hline 31 & Neutral Hand Soap & Innocuous & 6.50 & 0.585 \\
\hline 32 & Sun Lotion 3 & Innocuous & 7.19 & 0.580 \\
\hline 33 & Sun Baby Lotion & Innocuous & 8.73 & 0.606 \\
\hline
\end{tabular}

Continued on next page 
Table 3 - Continued from previous page

\begin{tabular}{|c|c|c|c|c|}
\hline Index & Material label & Type & $Z_{e}$ & $\rho_{e}$ \\
\hline 34 & Antiperspirant & Innocuous & 6.05 & 0.340 \\
35 & Beer 3 (Pale Ale) & Innocuous & 7.55 & 0.556 \\
36 & Olive Oil & Innocuous & 5.41 & 0.510 \\
37 & Alcohol Drink & Innocuous & 7.27 & 0.583 \\
38 & Rapeseed Oil & Innocuous & 5.40 & 0.516 \\
39 & Simple Eye Makeup Remover & Innocuous & 7.00 & 0.576 \\
40 & Body Lotion 2 & Innocuous & 6.92 & 0.563 \\
41 & Sugar & Innocuous & 6.49 & 0.524 \\
42 & Sun lotion 4 & Innocuous & 8.57 & 0.595 \\
43 & Hairspray 1 & Innocuous & 7.11 & 0.568 \\
44 & Beer 4 (IPA Ale) & Innocuous & 7.35 & 0.563 \\
45 & Beer 5anish Christmas Beer) & Innocuous & 7.42 & 0.560 \\
46 & Whiskey & Innocuous & 7.27 & 0.524 \\
47 & Beautifying Anti-Blemish Care & Innocuous & 7.15 & 0.582 \\
48 & Water & Innocuous & 7.05 & 0.562 \\
49 & Energy Drink & Innocuous & 7.10 & 0.586 \\
50 & Hairspray 2 & Innocuous & 8.10 & 0.391 \\
51 & Shaving Foam & Innocuous & 8.12 & 0.542 \\
52 & Toothpaste 1 & Innocuous & 8.19 & 0.665 \\
53 & Ammonia Nitrate & Threat & 6.73 & 0.490 \\
54 & Book & Innocuous & 9.74 & 0.419 \\
55 & C4 Simulant & Threat & 8.02 & 0.855 \\
56 & C4 & Threat & 6.44 & 0.497 \\
57 & Chocolate & Innocuous & 6.68 & 0.700 \\
58 & Comp B & Threat & 6.54 & 0.897 \\
59 & Crystal Sugar & Innocuous & 6.63 & 0.531 \\
60 & DVD Bomb Simulant & Threat & 7.49 & 0.878 \\
61 & Hard Cheese & Innocuous & 7.41 & 0.619 \\
62 & Marzipan & Innocuous & 6.47 & 0.648 \\
63 & Nitromethane & Threat & 6.86 & 0.575 \\
64 & PETN Simulant & Threat & 7.72 & 0.719 \\
65 & Peanut Butter & Innocuous & 6.94 & 0.636 \\
66 & PETN & Threat & 6.73 & 0.839 \\
67 & Shampoo & Innocuous & 7.37 & 0.582 \\
68 & Soap Bar & Innocuous & 6.13 & 0.595 \\
69 & Soft Cheese & Innocuous & 7.35 & 0.604 \\
70 & Sun Cream & Innocuous & 7.32 & 0.571 \\
71 & TNT (chunks) & Threat & 6.63 & 0.434 \\
72 & Toothpaste 2 & Innocuous & 8.26 & 0.678 \\
74 & Innocuous & 7.13 & 0.639 \\
\hline
\end{tabular}

\section{ACKNOWLEDGMENTS}

The author would like to thank Innovation Fund Denmark for funding the project.

\section{REFERENCES}

[1] Wells, K. and Bradley, D., "A review of X-ray explosives detection techniques for checked baggage," Applied Radiation and Isotopes 70(8), 1729-1746 (2012). 
[2] Brooks, R. A. and Di Chiro, G., "Beam hardening in x-ray reconstructive tomography," Physics in medicine Es biology 21(3), 390 (1976).

[3] De Man, B., Nuyts, J., Dupont, P., Marchal, G., and Suetens, P., "Metal streak artifacts in x-ray computed tomography: a simulation study," IEEE Transactions on Nuclear Science 46(3), 691-696 (1999).

[4] Alvarez, R. E. and Macovski, A., "Energy-selective reconstructions in x-ray computerised tomography," Physics in Medicine 83 Biology 21(5), 733 (1976).

[5] Ying, Z., Naidu, R., and Crawford, C. R., "Dual energy computed tomography for explosive detection," Journal of X-ray Science and Technology 14(4), 235-256 (2006).

[6] Azevedo, S. G., Martz, H. E., Aufderheide, M. B., Brown, W. D., Champley, K. M., Kallman, J. S., Roberson, G. P., Schneberk, D., Seetho, I. M., and Smith, J. A., "System-independent characterization of materials using dual-energy computed tomography," IEEE Transactions on Nuclear Science 63(1), 341-350 (2016).

[7] Champley, K. M., Azevedo, S. G., Seetho, I. M., Glenn, S. M., McMichael, L. D., Smith, J. A., Kallman, J. S., Brown, W. D., and Martz, H. E., "Method to extract system-independent material properties from Dual-Energy X-ray CT," Submitted to IEEE Transactions on Nuclear Science (2018) (2018).

[8] Silva, A. C., Morse, B. G., Hara, A. K., Paden, R. G., Hongo, N., and Pavlicek, W., "Dual-energy (spectral) CT: applications in abdominal imaging," Radiographics 31(4), 1031-1046 (2011).

[9] Shikhaliev, P. M., "Energy-resolved computed tomography: first experimental results," Physics in Medicine E Biology 53(20), 5595 (2008).

[10] Rebuffel, V., Rinkel, J., Tabary, J., and Verger, L., "New perspectives of X-ray techniques for explosive detection based on CdTe/CdZnTe spectrometric detectors," Proc. of the Int. Symp. on Digital Industrial Radiology and Computed Tomography 2 (2011).

[11] McCollough, C. H., Leng, S., Yu, L., and Fletcher, J. G., "Dual-and multi-energy CT: principles, technical approaches, and clinical applications," Radiology 276(3), 637-653 (2015).

[12] Anderson, N., Butler, A., Scott, N., Cook, N., Butzer, J., Schleich, N., Firsching, M., Grasset, R., De Ruiter, N., Campbell, M., et al., "Spectroscopic (multi-energy) CT distinguishes iodine and barium contrast material in mice," European radiology 20(9), 2126-2134 (2010).

[13] Fornaro, J., Leschka, S., Hibbeln, D., Butler, A., Anderson, N., Pache, G., Scheffel, H., Wildermuth, S., Alkadhi, H., and Stolzmann, P., "Dual-and multi-energy CT: approach to functional imaging," Insights into imaging 2(2), 149-159 (2011).

[14] Roessl, E. and Proksa, R., "K-edge imaging in x-ray computed tomography using multi-bin photon counting detectors," Physics in Medicine 63 Biology 52(15), 4679 (2007).

[15] Schlomka, J., Roessl, E., Dorscheid, R., Dill, S., Martens, G., Istel, T., Bäumer, C., Herrmann, C., Steadman, R., Zeitler, G., et al., "Experimental feasibility of multi-energy photon-counting k-edge imaging in pre-clinical computed tomography," Physics in Medicine $\mathscr{E}$ Biology 53(15), 4031 (2008).

[16] Busi, M., Mohan, K. A., Dooraghi, A. A., Champley, K. M., Olsen, U. L., and Martz, H. E., "Method for System-Independent Material Characterization from Spectral X-ray CT," Accepted by NDTESE International (2019).

[17] Swinehart, D., "The beer-lambert law," Journal of chemical education 39(7), 333 (1962).

[18] Dreier, E. S., Kehres, J., Khalil, M., Busi, M., Gu, Y., Feidenhans, R., and Olsen, U. L., "Spectral correction algorithm for multispectral CdTe x-ray detectors," Optical Engineering 57(5), 054117 (2018).

[19] Busi, M., Olsen, U. L., Knudsen, E. B., Frisvad, J. R., Kehres, J., Dreier, E. S., Khalil, M., and Haldrup, K., "Simulation tools for scattering corrections in spectrally resolved x-ray computed tomography using McXtrace," Optical Engineering 57(3), 037105 (2018).

[20] Champley, K., "Livermore tomography tools (LTT) technical manual, LLNL-SM-687016, pages 1-165, 2016,"

[21] Gorecki, A., Brambilla, A., Moulin, V., Gaborieau, E., Radisson, P., and Verger, L., "Comparing performances of a CdTe X-ray spectroscopic detector and an X-ray dual-energy sandwich detector," Journal of Instrumentation 8(11), P11011 (2013).

[22] Brambilla, A., Ouvrier-Buffet, P., Rinkel, J., Gonon, G., Boudou, C., and Verger, L., "CdTe linear pixel x-ray detector with enhanced spectrometric performance for high flux x-ray imaging," IEEE Transactions on Nuclear Science 59(4), 1552-1558 (2012). 
[23] Dooraghi, A. A., Fix, B. J., Smith, J. A., Brown, W. D., Azevedo, S. G., and Martz, H. E., "Characterization of a spectroscopic detector for application in x-ray computed tomography," in [Developments in X-Ray Tomography XI], 10391, 103911G, International Society for Optics and Photonics (2017).

[24] Eger, L., Do, S., Ishwar, P., Karl, W. C., and Pien, H., "A learning-based approach to explosives detection using multi-energy x-ray computed tomography," in [2011 IEEE International Conference on Acoustics, Speech and Signal Processing (ICASSP)], 2004-2007, IEEE (2011).

[25] Chantler, C. T., "Theoretical form factor, attenuation, and scattering tabulation for $\mathrm{Z}=1-92$ from $\mathrm{E}=1-10$ $\mathrm{eV}$ to $\mathrm{E}=0.4-1.0 \mathrm{MeV}$," Journal of Physical and Chemical Reference Data 24(1), 71-643 (1995).

[26] Haldrup, K., "Singular value decomposition as a tool for background corrections in time-resolved xfel scattering data," Philosophical Transactions of the Royal Society B: Biological Sciences 369(1647), 20130336 (2014). 